\title{
Vector Groups and the Equality Problem for Vector Addition Systems
}

\author{
By Michael Anshel
}

\begin{abstract}
Our purpose is to demonstrate that results concerning the equality problem for vector addition systems, may be uséd to establish the decidability and undecidability of decision problems associated with the class of $H N N$ extensions of the infinite cyclic group. We call these groups 'vector groups.'
\end{abstract}

By vector groups we understand the $H N N$ groups $G\left(p_{1}, q_{1}, \ldots, p_{k}, q_{k}\right)$ given by

$$
\left\langle a_{1}, \ldots, a_{k}, b ; a_{1}^{-1} b^{p_{1}} a_{1}=b^{q_{1}}, \ldots, a_{k}^{-1} b^{p_{k}} a_{k}=b^{q_{k}}\right\rangle,
$$

where the exponent pairs $p_{i}, q_{i}$ occurring in (I) are positive and relatively prime. In [2] the conjugacy problem for vector groups was reduced to the reachability problem for self-dual vector addition systems, and subsequently an algebraic solution of both problems was presented in [3]. Here we demonstrate that recent results concerning the equality problem for vector addition systems, having surprising algebraic consequences for vector groups.

Let $G$ be a vector group and call $m$ a conjugate power of $l$ in $G$ when $b^{m}=$ $x b^{l} x^{-1}, x$ in $G$ and a positive conjugate power if in addition $x$ is given by a positive word in the generators $a_{1}, \ldots, a_{k}, b$ of $G$ (i.e., one which involves no negative exponents). The set of (positive) conjugate powers of $l$ in $G$ is called a (positive) conjugate power set. Here we consider the question as to whether the (positive) conjugate powers of $l$ in $G_{1}$ and $G_{2}$ coincide where $G_{1}$ is given in (I) and $G_{2}$ arises from $G_{1}$ by removing a particular defining relation, $a_{i}^{-1} b^{p_{i}} a_{i}=b^{q_{i}}$. We will prove:

THEOREM A. It is decidable whether the removal of a particular defining relation from a vector group changes a conjugate power set.

THEOREM B. It is undecidable whether the removal of a particular defining relation from a vector group changes a positive conjugate power set.

For $G=G\left(p_{1}, q_{1}, \ldots, p_{k}, q_{k}\right)$ let $\mathrm{CP}(l, G)$ and $\operatorname{PCP}(l, G)$ denote respectively the conjugate and positive conjugate powers of $l$ on $G$. From [1, pp. 22-23], it follows that $m$ lies in $\mathrm{CP}(l, G)$ if and only if there is a sequence $l=l_{1}, \ldots, l_{n}=m$ such that $l_{i+1}=l_{i}\left(p_{j} / q_{j}\right)$ or $l_{i+1}=l_{i}\left(q_{j} / p_{j}\right)$. Similarly, $m$ lies in $\operatorname{PCP}(l, G)$ if and only if $l=l_{1}, \ldots, l_{n}=m$ where $l_{i+1}=l_{i}\left(p_{j} / q_{j}\right)$. These observations allow us to restrict our attention to those positive integers $l$ whose prime divisors are among the prime divisors of the exponents of $G$.

Received March 17, 1977.

AMS (MOS) subject classifications (1970). Primary 20E05, 20F05; Secondary 02G05, $68 \mathrm{~A} 30$.

Copyright (s) 1978, American Mathematical Society 
By a vector addition system (of dimension $n$ ) we mean a pair $(d, W), d$ in $N^{n}, W$ a finite subject of $Z^{n}$ (where $N$ and $Z$ denote the nonnegative integers and integers respectively). Let us call $d^{\prime}$ reachable in $(d, W)$ if $d=d^{\prime}$ or $d^{\prime}=d+w_{1}+\cdots+w_{t}$, $w_{i}$ in $W$ and $d+w_{1}+\cdots+w_{s}$ in $N^{n}, s=1, \ldots, t$.

Let $R(d, W)$ denote the reachable vectors in $(d, W)$ or reachability set of $(d, W)$. Let $K$ be a class of vector addition systems. By the equality problem for $K$ we mean the problem of deciding for arbitrary $\left(d_{1}, W_{1}\right),\left(d_{2}, W_{2}\right)$ in $K$ whether $R\left(d_{1}, W_{1}\right)=$ $R\left(d_{2}, W_{2}\right)$. The special equality problem is to decide the equality problem in those cases where $d_{1}=d_{2}$ and $W_{2}$ arises from $W_{1}$ by removal of a vector.

Let VAS denote the class of vector addition systems and SDVAS denote the self-dual vector addition systems $(d, W)$ defined by the property: $W=-W$ (i.e. $w$ in $W$ if and only if $-w$ in $W$ ).

LEMma 1. The problem of deciding whether the removal of a particular defining relation from a vector group changes a (positive) conjugate power set is reducible to the special equality problem for (VAS) SDVAS.

Proof. Let $G_{1}$ be as in (I) and $G_{2}$ arise from $G_{1}$ by removal of one of the defining relations $a_{i}^{-1} b^{p_{i}} a_{i}=b^{q_{i}}$. It follows from the remarks above that we need consider only those positive integers $l$ whose prime divisors are among $c_{1}, \ldots, c_{n}$, the prime divisors of the exponents of $G_{1}$. To obtain the indicated vector addition systems we take $d=\left(s_{1}, \ldots, s_{n}\right)$ and $w_{i}=\left(u_{1 i}, \ldots, u_{n i}\right)$

$$
\begin{aligned}
l & =c_{1}^{s_{1}} \cdots c_{n}^{s_{n}}, \\
p_{i} & =c_{1}^{e_{1 i}} \cdots c_{n}^{e_{n i}}, \quad q_{i}=c_{1}^{e_{1 i}^{\prime}} \ldots c_{n}^{e_{n i}^{\prime}}, \quad i=1, \ldots, k, \\
u_{j i} & = \begin{cases}e_{j i} & \text { if } e_{j i} \neq 0, \quad j=1, \ldots, n, \\
-e_{j i}^{\prime} & \text { otherwise, }\end{cases}
\end{aligned}
$$

(and note $e_{j i} \cdot e_{j}^{\prime}=0$ ).

Let $W_{1}^{\prime}$ consist of $w_{1}, \ldots, w_{k}, W_{2}^{\prime}=W_{1}^{\prime}-\left\langle w_{i}\right\rangle, w_{1}=W_{1}^{\prime} \cup-W_{1}^{\prime}$ and $w_{2}=$ $W_{2}^{\prime} \cup-W_{2}^{\prime}$.

Our lemma follows from the observation that for $i=1,2$ we have $m$ in $\mathrm{CP}\left(l, G_{i}\right)$ (respectively $\left.\operatorname{PCP}\left(l, G_{i}\right)\right)$ if and only if $d^{\prime}$ in $R\left(d, W_{i}\right)$ (respectively $R\left(d, W_{i}^{\prime}\right)$ ) where $d^{\prime}=\left(t_{i}, \ldots, t_{n}\right)$ and $m=c_{1}^{t_{1}} \cdots c_{n}^{t_{n}}$.

A set $L \subseteq N^{n}$ is said to be linear if and only if $L=R(d, W)$ where $W$ consists of nonnegative integral vectors. $L$ is said to be semilinear if and only if it is the finite union of a finite number of linear sets.

It has been pointed out to the author by Professor John Hopcroft that:

LEMMA 2. The reachability sets of self-dual vector addition systems are effectively computable semilinear sets.

Remark. Lemma 2 seems to be known to several workers in this area (cf. [4], [7]).

LEMma 3. The equality problem for SDVAS is decidable.

Lemma 3 is a consequence of the fact that semilinear sets in $N^{n}$ form an effec- 
tively computable Boolean algebra [5]. Extending a previous result of Michael Rabin, M. Hack proved [6] :

LEMMA 4. The special equality problem for VAS is undecidable.

Theorems A and B are now seen to be consequences of Lemmas 1, 3 and 4.

The author wishes to thank Professor John E. Hopcroft for making available [7].

Department of Computer Sciences

The City College of the City University of New York

New York, New York 10031

1. M. ANSHEL, "Conjugate powers in HNN groups," Proc. Amer. Math. Soc., v. 54, 1976, pp. 19-23.

2. M. ANSHEL, "Decision problems for HNN groups and vector addition systems," Math. Comp., v. 30, 1976, pp. 154-156.

3. M. ANSHEL, "The conjugacy problem for $H N N$ groups and the word problem for commutative semigroups," Proc. Amer. Math. Soc., v. 61, 1976, pp. 223-224.

4. E. W. CARDOZA, Computational Complexity of the Word Problem for Commutative Semigroups, MAC Technical Memo 67, Project MAC, M.I.T., Cambridge, Mass., October 1975.

5. S. GINSBURG, The Mathematical Theory of Context-Free Languages, McGraw-Hill, New York, 1966.

6. M. HACK, The Equality Problem for Vector Addition Systems, CSG Memo 121, Project MAC, M.I.T., Cambridge, Mass., April 1975. (Also to appear in J. Theoret. Comput. Sci.)

7. J. HOPCROFT \& J. J. PANSIOT, Decidability of Self-Dual Vector Addition Systems, Dept. of Comput. Sci., Cornell Univ., Ithaca, N. Y. 